\title{
TTR
}

Traduction, terminologie, re?daction

\section{Les barbarismes d'Otfrid de Wissembourg : traduction et exégèse vernaculaires en vieux-haut-allemand}

\section{Hans-Herbert S. Räkel}

Volume 2, numéro 1, 1er semestre 1989

Carrefours de la traduction

URI : https://id.erudit.org/iderudit/037035ar

DOI : https://doi.org/10.7202/037035ar

Aller au sommaire du numéro

Éditeur(s)

Association canadienne de traductologie

ISSN

0835-8443 (imprimé)

1708-2188 (numérique)

Découvrir la revue

Citer cet article

Räkel, H.-H. S. (1989). Les barbarismes d'Otfrid de Wissembourg : traduction et exégèse vernaculaires en vieux-haut-allemand. TTR, 2(1), 89-102.

https://doi.org/10.7202/037035ar

Tous droits réservés @ C TTR: traduction, terminologie, rédaction — Les auteurs, 1989 document est protégé par la loi sur le droit d'auteur. L’utilisation des services d'Érudit (y compris la reproduction) est assujettie à sa politique d'utilisation que vous pouvez consulter en ligne.

https://apropos.erudit.org/fr/usagers/politique-dutilisation/ 


\title{
Les barbarismes d'Otfrid de Wissembourg: traduction et exégèse vernaculaires en vieux-haut-allemand
}

\author{
Hans-Herbert S. Räkel
}

Lorsque Jacob Grimm et ses contemporains se penchèrent sur l'histoire de la langue allemande - nous sommes à l'époque napoléonienne - , c'était dans le but de retrouver les racines d'une civilisation en état de crise. Le fondateur de la philologie germanique se garda toutefois de valoriser trop le passé au détriment du présent, et s'il admirait la beauté et l'économie de la langue gothique, il ne sous-estimait pas pour autant la richesse et la diversification des moyens d'expression de l'allemand moderne ${ }^{1}$ sous la plume d'un Gothe, son contemporain. Ses successeurs allaient par contre privilégier presque exclusivement la recherche des racines, ces signifiants rudimentaires rattachés à ce que Franz Bopp appelait «Urbegriffe» ou concepts primitifs (1868, p. III). Malgré l'affirmation grandissante de sa scientificité, la recherche des racines conservera cette nostalgie de vérité qui est le fond de toute étymologie; to etymon ne signifie-t-il pas "le vrai»! N'est-il pas tentant de chercher dans le sémantème le sens, et dans l'étymon un message sensé, une révélation? Or, les premiers textes de littérature allemande, qui semblent si bien se prêter aux besoins de la linguistique historique, ne sont-ils pas dominés par un autre souci que celui de leur passé? Le premier livre allemand est un dictionnaire ${ }^{2}$ qui essaie de rendre compte en allemand d'un vocabulaire latin difficile, en partie archaïque, et ce n'est en effet pas le mystère des racines qui préoccupe les

1. Jacob Grimm (1864, p. 281): «Halten wir die gothische sprache des vierten jh. gegen unsre heutige, dort ist wollaut und schöne behendigkeit, hier, auf kosten jener, vielfach gesteigerte ausbildung der rede.»

2. D'après le premier mot de ce dictionnaire on lui a donné le titre «ABROGANS». Le manuscrit a été écrit vers la fin du $8^{\mathrm{e}}$ siècle dans le Sud-Ouest de l'Allemagne et est conservé à la Bibliothèque conventuelle de St-Gall. 
germanophones du temps de Charlemagne, mais l'art de la greffe: faire croître et fructifier la culture latine et chrétienne sur une souche barbare, voilà l'objet d'un effort culturel conscient et assidu. S'il est vrai que l'on reconnaît l'arbre à ses fruits, c'est bien le greffage, processus par lequel les dialectes germaniques parlés dans l'Empire de Charlemagne se mettront à assimiler la latinité chrétienne, qui tiendra lieu d'origine à la langue allemande, de sorte qu'il ne serait pas faux de dire qu'elle est le produit d'un vaste travail de traduction ${ }^{3}$.

Otfrid de Wissembourg, l'auteur de l'œuvre la plus importante de la littérature en vieux-haut-allemand, le Livre des Évangiles, achevé entre 863 et 871 , vraisemblablement dès $865^{4}$, a accompagné son travail d'écriture d'une profonde réflexion. Il nous en livre de grands fragments dans une préface et trois dédicaces dont l'une, adressée à l'archevêque de Mayence, Liutbert, est écrite en latin ${ }^{5}$. Il y dit:

scripsi ... evangeliorum partem franzisce compositam, interdum spiritalia moraliaque verba permiscens. (23)

L'intention de l'auteur fut donc de composer, dans la langue des Francs, le récit de la vie du Christ, en s'attribuant à lui-même le rôle du narrateur qui non seulement donne une forme poétique à sa narration, mais y ajoute d'importants passages réservés à l'exégèse des événements racontés. Si l'on a pu mettre en doute, notamment au $19 \mathrm{e}$ siècle, la qualité poétique de l'œuvre et le génie créateur de l'auteur, la recherche littéraire a néanmoins abouti à un consensus pour considérer le Livre des Évangiles comme une épopée biblique, ancrée dans la tradition chrétienne, portée par des besoins théologiques de son époque et animée par le souffle puissant de la compétence littéraire et de la foi de son auteur ${ }^{6}$.

En d'autres termes, on peut affirmer avec Dieter Wunder (1975, p. 23) que dans la littérature en vieux-haut-allemand «le Livre des

3. Introduction succincte en français: Franziska Raynaud (1982).

4. On trouve une synthèse des connaissances sur l'auteur et l'œuvre dans l'article «Otfrid von Weißenburg» de Werner Schröder (1987).

5. Le texte de la dédicace latine se trouve dans l'édition Erdmann (1965) aux pages 4 à 7 . Une édition critique avec un bref commentaire et une nouvelle traduction allemande a été publiée par Fidel Rädle (1974). Je cite le texte de cette dernière édition tout en donnant les numéros de lignes d'après l'édition Erdmann, d'accès plus facile. L'étude de Francis P. Magoun, Jr. (1943) contient une traduction anglaise, dépassée par les recherches récentes, mais reprise telle quelle lors de la réimpression de l'article (en langue allemande) par Wolfgang Kleiber (1978 éd.), pp. 74-108. La dédicace a fait l'objet d'une importante étude littéraire par Wiebke Freytag (1982), qui la situe dans la tradition épistolaire de son temps.

6. Cf. l'important ouvrage de Dieter Kartschoke (1975), qui a réuni une série de jugements négatifs formulés par les germanistes du $19^{e}$ siècle (p. 336 et sq.). 
Évangiles d'Otfrid est le seul texte d'envergure qui ne soit pas une traduction». ${ }^{7}$

Ceci revient à dire que le texte d'Otfrid est indéniablement le produit d'une compétence linguistique représentative et respectueuse des normes du vieux-haut-allemand, voire du dialecte francique rhénan méridional parlé par l'auteur, et que tout en s'attachant aux événements et au contenu de ses sources, il s'est parfaitement émancipé de l'emprise du latin, notamment en ce qui concerne la syntaxe.

Or, dans sa préface latine, Otfrid note qu'il n'a pas toujours évité les «barbarismes» et les «solécismes» et qu'il lui est arrivé d'enfreindre les règles de la grammaire latine en écrivant sa langue francique. Dans son analyse de cette préface, Rainer Patzlaff (1975, p. 58) écrit sans hésitation: «Otfrid parle des difficultés d'un traducteur," ${ }^{8}$ et Hartmut Günther (1985, p. 43), dans une étude récente qui traite plus particulièrement du statut théorique de la langue écrite, constate qu'Otfrid semble s'attaquer à des problèmes plutôt secondaires en négligeant les "véritables" problèmes de l'écriture et de la traduction.

Est-il concevable qu'Otfrid se soit considéré comme traducteur, tout en voulant égaler non seulement les poètes chrétiens de la basse antiquité mais encore les «gentilium vates» (13)?

Otfrid regrette en effet que les Francs n'aient pas suivi l'exemple des auteurs chrétiens tels que Juvencus, Arator, Prudentius qui ont "rehaussé avec goût dans leur langue les paroles et les miracles du Christ» - «sua lingua dicta et miracula Christi decenter ornabant» (18-19) - , et ceci bien que les Francs aient été instruits de la même foi et de la même grâce que leurs éminents prédécesseurs. Otfrid veut relever ce défi pour enfin «dire dans sa propre langue la splendeur glorieuse des paroles divines» (20) - leur «splendeur» et non les paroles elles-mêmes, tout comme les auteurs cités qui en ont fait des paraphrases poétiques. Il y a toutefois une différence importante entre Otfrid et les auteurs dont il vient d'être question: leurs lecteurs pouvaient lire les évangiles en latin. En lisant les paraphrases poétiques, ils procédaient à une sorte de célébration de l'Évangile dont le texte authentique restait la base. Or, Otfrid se rend bien compte de sa position particulière lorsqu'il indique un peu plus loin un autre but de son travail: pour que celui qui craint la difficulté d'une langue étrangère

7. Chez Wunder, cette constatation est importante d'un point de vue méthodologique, puisqu'il cherche à élucider la forme et la fonction de la proposition subordonnée en allemand; il doit donc s'assurer que son corpus est bel et bien représentatif de la langue étudiée, sans présenter d'interférences systématiques ou occasionnelles avec le latin. L'opinion de Wunder reflète un consensus général de la recherche.

8. Ulrich Ernst (1975, p. 129 et sq.) constate également chez Otfrid un intérêt marqué pour la théorie de la traduction, dans la tradition de saint Jérôme et d'Agobard de Lyon. 
puisse connaître ici par l'intermédiaire de sa propre langue les paroles saintes et comprendre dans sa langue la loi divine. (25-28) N'est-ce pas là le but d'une traduction? Otfrid semble donc délibérément fondre ce que nous distinguons: traduction fidèle - «imitative» ${ }^{9}$ - et célébration poétique (ou paraphrase) du texte original. Il attribue à son œuvre le pouvoir d'élever l'âme au-dessus du monde auquel la relient les cinq sens, convaincu que la profondeur du cour retiendra pour toujours dans la mémoire ces lectures rédigées en langage vernaculaire («theotisce conscriptas", 56). Dans la terminologie nouvelle de $\mathrm{K}$. Reiß et H.J. Vermeer (1984, p. 124 et sq.), qui distinguent équivalence et adéquation ${ }^{10}$, Otfrid opère un transfert de la parole divine dans la langue des Francs, une "translation", et même une "traduction", puisqu'il part de textes écrits en latin.

La portée théologique, qui donne au Livre des Évangiles sa cohérence intratextuelle et intertextuelle, forme toutefois un contraste saisissant avec les préoccupations linguistiques qui pourront paraitre secondaires, mais auxquelles Otfrid semble accorder beaucoup d'importance, telle la transcription de sons allemands par l'alphabet latin ${ }^{11}$. Je n'aborderai pas ici la position de l'auteur face à ces questions; je préfêre analyser deux points précis qui ont été commentés par Otfrid, et qui sont susceptibles de nous éclairer sur la manière dont il conçoit le transfert qu'il entend réaliser à travers son écriture: il s'agit de l'expression de la négation et de l'usage du nombre et du genre des noms.

\section{Otfrid fait le commentaire suivant:}

Tandis que deux négations confirment en latin l'énoncé d'un discours, elles ont dans l'usage de cette langue presque toujours une signification négative, et bien que j'eusse pu éviter cela à l'occasion je me suis appliqué d'écrire, en raison de l'usage quotidien, comme on parle ordinairement. (93-96)

Cette remarque distingue deux niveaux, le premier de nature théorique, le deuxième de nature pratique. Otfrid a en effet observé un fait de langue en remarquant que le latin et le vieux-haut-allemand expriment par des signifiants différents (et, dans un certain sens, même contradictoires) un signifié équivalent voire identique. L'approche contrastive de l'auteur se situe de ce fait au niveau de la langue, non pas à celui de la parole. On s'étonnera même qu'Otfrid s'abstienne

9. Ce terme est emprunté à l'ouvrage fondamental Grundlegung einer allgemeinen Translationstheorie de Katharina Reiß et Hans J. Vermeer (1984, p. 93 et sq.).

10. Cf. également l'article de Katharina Reiß (1984).

11. Hartmut Günther (1985) place cette problématique dans un contexte plus vaste en mettant l'accent sur le statut propre du langage écrit et en s'élevant contre les «dogmes» saussuriens de la prédominance théorique de la 'langue' sur la 'parole' et de la priorité du langage parlé sur le langage écrit dans la recherche linguistique. 
ici de juger cette divergence puisque le prestige du latin s'impose et que l'usage allemand souffre d'une carence logique évidente. De cette remarque contrastive, Otfrid tire une conclusion pratique pour ce que nous appelons la traduction: il informe son lecteur de sa décision de respecter l'usage, c'est-à-dire le système et les normes du dialecte francique.

Certes on pourrait dire que la pratique de la traduction favorise de telles observations contrastives et aide à formuler des règles grammaticales précises, notamment dans le cas d'une langue comme le vieuxhaut-allemand qui, à l'époque de notre auteur, n'avait pas encore bénéficié d'une description grammaticale. Cependant nous nous refusons à ne voir dans cette réflexion qu'une systématisation théorique d'un phénomène de traduction. Théoriquement parlant, Otfrid adopte le point de vue de la linguistique contrastive dans le sens d'Eugenio Coseriu $^{12}$ (1972).

La pratique d'écriture de notre auteur le confirme. Conformément à la règle constatée, il écrira:

in guates nio $n i$ wangta (II, 10, 6)

«le salut ne leur fit jamais défaut"

où les deux particules de négation nio, ni se confirment et se renforcent mutuellement au lieu de s'annuler. Par contre, dans l'explication du miracle des noces de Cana, où Otfrid avait lu chez Alcuin:

Vinum ... non ex nihilo facere voluit

et où il traduit:

$N i$ wolt er fon niawihti... then selbon win wirken (II,10),

les deux négations s'annulent comme en latin. Nous pouvons conclure qu'Otfrid se comporte, lorsqu'il traduit, de façon tout à fait pragmatique, en respectant l'usage quotidien même lorsqu'il semble contrevenir à la règle qu'il a lui-même essayé de formuler. D'ailleurs, cette règle demanderait à être précisée. ${ }^{13}$

Revenons au problème principal, à cette apparente contradiction entre le projet littéraire et les préoccupations de détail, entre le rôle du narrateur-exégète et celui du traducteur, rôles qu'Otfrid semble vouloir concilier.

12. Eugenio Coseriu (1972) et (1981, conférence de 1976 réimprimée, p. 29). Reiß et Vermeer $(1984$, p. 142) insistent sur cette même distinction.

13. On peut consulter à ce sujet le chapitre 14 *Die Negation*, de Hermann Paul /

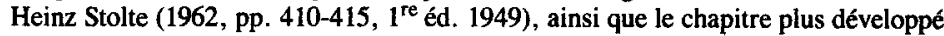
d'Ingeborg Schröbler (1975). L'introduction la plus pertinente aux problèmes logiques reflétés par la syntaxe allemande moderne a été donnée par Jean-Marie Zemb (1968). 
Dans le deuxième chapitre intitulé INVOCATIO SCRIPTORIS AD DEUM, c'est de toute évidence le narrateur-exégète qui parle. Otfrid ne prie pas Dieu de lui donner force et savoir pour accomplir une bonne traduction de ses sources, mais il invoque Dieu pour qu'il lui donne l'inspiration et qu'il parle par sa bouche, exactement comme il le fit en parlant par la bouche des prophètes et des apôtres:

Oh mon seigneur, je suis ton serviteur, ma pauvre mère est ta servante!

Pose ton doigt sur ma bouche, approche ta main de ma langue pour que je chante tes louanges.

Ce n'est pas un hasard si l'auteur parle de sa bouche et non de sa plume, s'il se substitue au psalmiste en chantant ces paroles:

$O$ domine, quia ego servus tuus et filius ancillae tuae

(psaume 115, 16)

Domine, labia mea aperies et os meum annuntiabit laudem tuam

(psaume 50, 17),

s'il fait allusion au miracle du Christ qui avait ouvert les oreilles et délié la langue du sourd-muet (Marc 7, 33) et s'il demande au «seigneur de toutes les langues» de composer ses mots:

thu druhtin rihti wort min

(I, 2, 32).

Nous constatons, sans aller plus loin dans cette analyse, que l'auteur n'entend absolument pas faire du mot-à-mot en annonçant à son tour la bonne nouvelle, en célébrant les paroles et les miracles du Christ. (I, 2, 8-9)

Pourtant, si nous revenons à la dédicace latine, nous sommes déconcertés par des considérations d'un tout autre ordre: l'auteur ne se contente plus de constater un décalage comme dans le cas de la négation, il va jusqu'à s'accuser; nous sommes loin du discours de la parole divine dont Otfrid se disait l'instrument dans l'invocation que nous venons d'analyser.

Interdum enim masculinum latinae linguae in hac feminino protuli, et cetera genera necessarie simili modo permiscui; numerum pluralem singulari, singularem plurali variavi, et tali modo in barbarismum et solocismum saepius coactus incidi. (98-101)

«Par ailleurs j'ai traduit le masculin de la langue latine par un féminin dans celle-ci, et de la même manière j'ai nécessairement mélangé les autres genres; j'ai rendu un pluriel par un singulier et un singulier par un pluriel. Ainsi j'ai été contraint de commettre des barbarismes et des solécismes.»

Il y a quelque chose de troublant dans cet aveu: notre auteur, qui par ailleurs a décidé de suivre l'usage commun du francique pour 
la négation, et ce contrairement aux règles latines, semble attribuer ici un tel prestige à cette langue qu'il se voit obligé de considérer comme fautif le genre des noms en francique lorsque celui-ci differe du latin! Mais cette fois encore il ne va pas "corriger" le francique, d'autant moins qu'il est conscient que les fautes commises sont le produit d'une certaine nécessité et qu'il les commet sous l'effet d'une contrainte. Bien que formulé en termes de difficulté de traduction, le point de vue est encore celui de l'analyse contrastive des deux langues, comme ce fut le cas dans le traitement de la négation, point de vue confirmé un peu plus loin par l'affirmation curieuse qui insiste encore une fois sur le caractère «fautif» du francique:

Stupent in aliis vel litterula parva artem transgredi, et pene propria lingua vitium generat per singula verba. (112-113)

«Ils s'offusquent d'enfreindre les règles d'une langue étrangère dans le moindre détail, tandis que leur propre langue ne se gêne à peine de produire des fautes dans chaque mot.»

C'est la langue qui génère des fautes, non pas l'écrivain et encore moins le traducteur, qui lui au moins en est conscient.

D'un point de vue strictement linguistique, cette idée est aberrante. Aussi les commentateurs de la préface se sont-ils en général refusés à prendre ce texte au pied de la lettre, préférant y voir une signification atténuée ou même imagée qui désamorcerait le reproche. Je pense que l'analyse de Rainer Patzlaff ${ }^{14}$ (1975) a le mérite d'avoir éliminé plusieurs malentendus à ce sujet et qu'elle permet d'aller plus loin dans la réflexion sur le statut du texte. Les auteurs cités par Patzlaff avaient en effet essayé de comprendre «barbarisme et solécisme» comme une sorte de figure de style signifiant «un certain manque, une certaine infériorité de la langue par rapport au latin". Une telle interprétation est ancienne et bien établie, puisque depuis Tertullien le christianisme oppose dans un but apologétique le contenu religieux s'exprimant avec humilité à la forme soignée et brillante mais futile de la littérature païenne. Ulrich Ernst (1975, p. 125 et sq.) a esquissé cette tradition en étudiant le Livre des Évangiles, tradition qui en fin de compte fera des défauts formels le signe même de l'humilité, comme semblait le suggérer saint Augustin lorsqu'il écrivit:

Utrum enim Ignoscere producta an correpta tertia syllaba dicatur, non multum curat qui peccatis suis Deum ut ignoscat petit, quolibet modo illud verbum sonare potuerit.

«Celui qui prie Dieu de ne pas retenir ses péchés ne se tourmentera pas beaucoup de savoir si la troisième syllabe du verbe ignoscere se prononce ou ne se prononce pas.»

14. Patzlaff (1975) commente les termes 'barbarisme' et 'solécisme' (pp. 53 à 59 de l'ouvrage cité). 
Au-delà de ce contexte, qu'Otfrid n'a pu ignorer, il faut pourtant admettre avec Patzlaff que l'auteur utilise les termes de «barbarisme» et de «solécisme» en toute connaissance de cause. En effet, la substitution du genre d'un nom par un autre est bel et bien un barbarisme, parce qu'il apparaît dans une seule partie du discours; une confusion du nombre par contre est un solécisme, parce que la faute affecte la syntaxe. Patzlaff a montré de façon tout à fait convaincante que la phrase d'Otfrid répond parfaitement à ces définitions, puisque la première partie est une description du barbarisme (confusion de genres: «... genera ... permiscui»), la deuxième celle du solécisme (confusion de nombres: «numerum ... variavi»); la conclusion va jusqu'à citer ces termes mêmes dans l'ordre où ils ont été évoqués: «in barbarismum et solecismum ... incidi».

Même si dès l'époque de Donat, au $4^{\mathrm{e}}$ siècle, nombreux sont ceux qui confondent ces notions («multi errant qui putant etiam in una parte orationis fieri solœecismum») et même si la locution «barbarismus et solœcismus» est devenue l'expression habituelle pour désigner les défauts d'un texte mal écrit, le sens propre de ces termes reste connu et est enseigné, par exemple chez Isidore:

Solœcismus est plurimorum verborum inter se inconveniens compositio, sicut barbarismus unius verbi corruptio. (I, 33)

Nous sommes donc bien obligés de reconnaître avec Patzlaff qu'Otfrid utilise ces termes au sens propre. On a pensé que cette autocritique ne portait que sur les mots d'emprunt, comme lat. crux (f.) devenu en vha. cruzi (n.), ou sur les cas d'hésitation en vieux-haut-allemand. Mais cela arrive trop rarement pour que notre auteur ait pu en faire un sujet de réflexion aussi fondamental.

Faut-il alors chercher à l'excuser? C'est ce que Patzlaff (1975, p. 58) semble vouloir faire: «Il ne faut pas oublier qu'Otfrid écrivait à une époque où la tradition de la version interlinéaire était encore vivante (...) Otfrid se distancie consciemment de telles traductions pédantes qui collent à leur modèle, pour respecter en traduisant la propriété de la langue francique et pour écrire du bon francique.» C'est ce qui expliquerait qu'Otfrid cherche à se défendre de ne pas respecter le texte sacré. Patzlaff ne donne toutefois aucun témoignage susceptible d'appuyer cette explication si ce n'est l'exemple de Martin Luther, qui, sept siècles plus tard, aurait à répondre à la même accusation. Contrairement à cet illustre successeur, qui a jugé que l'attaque était la meilleure défense (Sendbrief vom Dolmetschen), ${ }^{15}$ Otfrid aurait choisi une défense discrète mais habile en faisant valoir

15. Le texte du 'Sendbrief' a été reproduit dans: Hans Joachim Störig (1969, pp. 14-32). Le ton archaïsant de ce texte ne doit toutefois pas faire oublier qu'il s'agit d'une version modernisée. Pour une version critique on consultera l'édition Clemen (1912, tome IV, pp. 179-193). 
les propriétés de la langue francique et en prévenant les accusations par un aveu. Ce serait alors un autre exemple de cette modestie rhétorique qu'affectionnent, à tort ou à raison, les auteurs du moyen âge.

Cette explication suppose évidemment que la traduction dite interlinéaire ait été, à l'époque d'Otfrid, une pratique reconnue, dont les normes auraient pu empêcher «barbarismes et solécismes» au sens otfridéen. Or, cela est loin d'être certain. Même dans les versions interlinéaires, le genre des mots fait corps avec le lexème: ${ }^{16}$

adiutorium (n.) - helfa (f.),

le nombre ne semble pas protégé:

mella (pl.) - honec (sg.)

et assez régulièrement le cas de l'adjectif épithète suit celui de son substantif sans égard pour le «solécisme»:

ad vitam (f., acc.) perpetuam (f., acc.) - ze libe (m., dat.) euuikemu (m., dat.).

Mais il peut arriver que la version interlinéaire s'écarte de l'usage quotidien à seule fin de rendre compte de l'analyse grammaticale. Pour ce qui est de l'ordre des mots, l'écart par rapport à l'usage est habituel, mais cette pratique n'est pas dictée par l'autorité théologique du texte, autorité contre laquelle l'auteur Otfrid aurait pu se révolter pour s'excuser ensuite de sa témérité. La traduction interlinéaire trouve sa seule justification dans son but propre: celui de faire accéder le lecteur au texte même, avec lequel elle continue à faire corps dans une seule et même lecture. N'oublions pas que, dès le $8 \mathrm{e}$ siècle, il avait été possible de produire une traduction répondant parfaitement aux normes du concept moderne de «traduction imitative» (cf. note 9): le «Contra Judaeos» d'Isidore de Séville en vieux-haut-allemand ${ }^{17}$. Même s'il s'agit là d'une exception, elle devrait nous faire réfléchir avant que nous puissions investir la version interlinéaire d'une autorité et d'un sens profond qui va bien au-delà de sa fonction première et visible.

Nous devons reconsidérer le soi-disant scandale des barbarismes et des solécismes d'Otfrid: il n'est pas vraisemblable que cet auteur, formé par les plus grands maîtres du temps, notamment Hraban Maur, lui-même théologien, commentateur biblique et enseignant, ait utilisé ces termes de la grammaire latine pour critiquer ce que nous appelons

16. Les exemples sont empruntés à la version interlinéaire de la Règle de saint Benoit, du début du $9^{e}$ siècle: Ursula Daab (1959, pp. 7-8).

17. Édition: Hans Eggers (1964). Je cite au sujet de cette traduction Stefan Sonderegger $(1974$, p. 102), qui reconnaît à cette prose «une étonnante maitrise de la langue en ce qui concerne la précision de la traduction». 
une traduction. Il suffit de se rappeler qu'Otfrid écrit en vers allemands, qu'il reformule la narration à sa manière, qu'il insère dans la narration des éléments en vue de l'exégèse pour comprendre que le reproche de s'être écarté du texte sacré s'appliquerait non seulement au genre et au nombre des noms, mais bien plus au lexique, à la syntaxe et même au contenu du Livre des Évangiles.

Cette préface est claire: Otfrid, il nous faut l'admettre, est convaincu de l'infériorité intrinsèque de sa langue; il est persuadé que créer une oeuvre littéraire dans cette langue est une tâche impossible et il ne doute pas un instant de la supériorité et de la priorité des trois langues "nobles». ${ }^{18}$ Otfrid admet donc la discrimination de certaines langues par rapport à d'autres en fonction de leur structure, de leur degré de perfectionnement et à titre de moyen d'expression philosophique et instrument d'érudition. Une telle théorie linguistique contrastive s'impose au sein d'une civilisation où la langue n'a pas acquis le statut de symbole d'identification sociale et nationale dont les temps modernes l'ont investie. Mais, si c'est alors vraiment la langue qui produit les "fautes» - et c'est bien le jugement que l'auteur porte sur elle -, il faut replacer ce jugement dans le contexte exposé par l'auteur lui-même et non dans celui d'une hypothétique évolution qui aurait fait passer l'écriture des premiers balbutiements interlinéaires à la maîtrise de la "traduction". Ce contexte authentique est théologique et stipule que Dieu veut être adoré par toutes ses créatures:

Est tamen conveniens, ut qualicumque modo - sive corrupta seu lingua integrae artis - humanum genus auctorem omnium laudent, ... qui non verborum adulationem politorum, sed quaerit in nobis pium cogitationis affectum. (117-122)

Élargi, ce contexte admet en effet deux positions en apparence contradictoires: s'il est possible, normal, souhaitable d'honorer et de cultiver les langues qui ont été les premières à accueillir et à disperser la révélation, il n'en est pas moins naturel que les autres langues entretiennent avec Dieu une relation directe, non médiatisée. La révélation, voire son contenu, peut être versée dans tous les "récipients", quels qu'ils soient, ce que le miracle de la Pentecôte a fait comprendre avant qu'aucun texte évangélique n'ait été écrit ou traduit; mais cette révélation ne saurait être thésaurisée dans la matérialité du langage: elle ne paraîtra que par la voix même de Dieu. C'est à ce niveau-ci qu'Otfrid se considérera comme «traducteur», traducteur de la voix de Dieu, de l'esprit, non d'un texte. L'idée même de la traduction linguistique devient alors un non-sens. Otfrid renverse le modèle tripartite du lire, comprendre, traduire: Dieu doit «traduire», c'est-à-dire transmettre la foi à celui qui se trouvera alors en mesure de comprendre ce qu'il va lire. Cette théorie spirituelle de la traduction est diamétrale-

18. Otfrid écrit «edilzungun* (I, 1, 53). 
ment opposée à l'idée païenne d'un sens (pouvoir) attaché à la matérialité du langage. On peut rappeler ici que, à l'époque même d'Otfrid, la piété populaire des tribus germaniques christianisées restait très attachée à la magie de la parole. Les formules magiques et les incantations qui ont été conservées nous montrent que le Christ avait remplacé les dieux païens pour guérir les maladies des humains comme celles des animaux. ${ }^{19}$ Or, traduire un texte en respectant religieusement les «mots" (verbum), voire la «lettre» (Luther: "Buchstabe"), c'est croire en la magie de la formule, c'est considérer l'esprit comme prisonnier de la lettre. D'un point de vue purement théologique, une telle «traduction» ferait preuve d'orgueil puisqu'elle attend plus du travail de transposition et d'expression ainsi que du pouvoir d'exégèse que de l'inspiration divine. Un traducteur qui procéderait ainsi manquerait de foi, puisqu'il croirait que le sens est donné avec le texte et qu'il ne pourrait concevoir qu'on doive l'y mettre avec l'aide de Dieu.

Faire d'Otfrid un traducteur inspiré, c'est lui attribuer le rôle de poète qui célèbre l'Évangile en le racontant, non point pour rendre en allemand un texte latin, mais pour inviter son lecteur à y apporter sa foi. Puisque c'est là le but de cette écriture, le langage en tant que tel se trouve relativisé - aussi bien d'ailleurs que le contenu de la narration. Il devient dès lors possible et même nécessaire de le modifier si cela permet de mieux connaître la vérité. Ainsi Otfrid ajoute par exemple à la narration des noces de Cana une introduction dans laquelle il mentionne la fiancée et la joie des époux d'avoir Jésus auprès d'eux. ${ }^{20} \mathrm{Ce}$ détail facilitera l'exégèse puisque le Christ, qui

19. Les principales formules magiques de la période du vieux-haut-allemand ont été publiées, avec une traduction en allemand moderne, dans l'anthologie de Horst Dieter Schlosser (1970). Voici la traduction française de deux de ces formules, l'une païenne, l'autre chrétienne:

Phol et Wodan se rendaient au bois. C'est alors que le jeune étalon de Balder se tordit un pied. Sinthgunt prononça une prière magique ainsi que Sunna, sa sœur; Frija prononça une prière magique ainsi que Volla, sa sœur; Wodan prononça une prière magique dont lui seul connaît le charme:

«fracture d'os, épanchement de sang, foulure de membre:

os contre os, sang contre sang,

membre contre membre, comme figés.»

Le Christ et saint Étienne se rendaient à la ville de Salonie. C'est alors que le cheval de saint Étienne fut pris d'une maladie. À l'exemple du Christ guérissant la maladie du cheval de saint Étienne, moi, je guéris, avec l'aide du Christ, la maladie de ce cheval-ci. Pater noster...

20. Michel et Schwarz (1978, p. 125) ont forgé le terme heureux de «Sinndruck» pour analyser ce processus: «Nicht das [allegorische] Designat wird erklärt, sondern das [allegorische] Designans in seiner Legitimität vom Sinndruck des eruierten Designats her.» 
est la joie, deviendra l'époux de sa fiancée l'Église! ${ }^{21}$ Nous refusons une telle manipulation, mais, du point de vue d'Otfrid, elle est souhaitable et nécessaire: à quoi bon raconter l'histoire des noces de Cana si ce n'est pour la "remplir» de son sens?

«Applique-toi toujours de bien remplir l'écriture!»

Thaz giscrip in rihti irfulli thu io mit mahti! (II, 9, 91)

C'est dire qu'une théorie de la traduction qui défend le «primat du but» 22 permet de rendre compte du statut ambigu de l'épopée biblique d'Otfrid dans la mesure où elle stipule que le «sens» appartient beaucoup moins au texte qu'au traducteur.

Université de Montréal

\section{Références}

BischofF, Bernhard, Johannes Duft et Stefan Sonderegger (1977). Die «Abrogans» - Handschrift der Stiftsbibliothek St. Gallen. St-Gall, Zollikofer Fachverlag AG.

Bopp, Franz (1868). Vergleichende Grammatik des Sanskrit, Send, Armenischen, Griechischen, Lateinischen, Litauischen, Altslavischen, Gothischen und Deutschen. $3 \mathrm{e}$ édition, Berlin, Ferd. Dümmler's Verlagsbuchhandlung.

Clemen, Otto (éd., 1912; 6e éd. 1967). Luthers Werke. Berlin, De Gruyter.

Coseriu, Eugenio (1981), «Falsche und richtige Fragestellungen in der Übersetzungstheorie», dans WILSS, Wolfram, éd. Übersetzungswissenschaft. Darmstadt, Wissenschaftliche Buchgesellschaft, Wege der Forschung 535, pp. 27-47;

21. La narration des noces de Cana - est primordiale pour la compréhension des bases théologiques et litteraires d'Otfrid et des exégètes de son entourage. Depuis environ deux décennies d'excellentes études ont contribué à une compréhension nouvelle de l'œuvre d'Otfrid en partant de cet épisode significatif, comme celles de Paul Michel et Alexander Schwarz (1978), Ute Schwab (1980) et Hartmut Freytag (1980).

22. Katharina Reiß et Hans J. Vermeer (1984, p. 95 et sq.) en ont fait la clef de voûte de leur théorie générale de la «translation*, franchissant ainsi une limite presque généralement respectée ou contournée jusqu'ici. Eugenio Coseriu (1981, p. 46) avait fait une remarque tout à fait pertinente dans le même sens: «Das Übersetzen ist am ehesten dem Sprechen analog, und es gelten deshalb für das Übersetzen wie für das Sprechen nur finalistisch motivierte und finalistisch differenzierte Normen. Auch die 'beste Übersetzung' schlechthin für einen bestimmten Text gibt es aus demselben Grund nicht: Es gibt nur die beste Übersetzung dieses Textes für bestimmte Adressaten, zu einem bestimmten Zweck und in einer bestimmten geschichtlichen Situation.» 
(1972). «Über Leistung und Grenzen der kontrastiven Grammatik», dans NICKEL, Gerhard (1972). Reader zur kontrastiven Linguistik. Francfort, Athenäum Fischer, pp. 39-57.

DaAB, Ursula (éd., 1959). Die Althochdeutsche Benediktinerregel des Cod. Sang 916. Tübingen, Niemeyer, Altdeutsche Textbibliothek 50.

EGGERS, Hans (1964). Der althochdeutsche Isidor. Tübingen, Niemeyer, Altdeutsche Textbibliothek 63.

ERdmann, Oskar ( $5^{e}$ éd., 1965). Otfrids Evangelienbuch. Tübingen, Niemeyer, Altdeutsche Textbibliothek 49.

ERNST, Ulrich (1975). Der Liber Evangeliorum Otfrids von Weißenburg Literarästhetik und Verstechnik im Lichte der Tradition. Cologne et Vienne, Böhlau.

FREYTAG, Wiebke (1982). "Otfrieds Briefvorrede "AD LIUTBERTUM" und die Accessus ad auctores», dans Zeitschrifi für deutsches Altertum, vol. 111, pp. 168-193.

FreYTAG, Hartmut (1980). «Liturgisches in Otfrieds Deutung der Hochzeit zu Kana», dans Zeitschrift für deutsches Altertum, vol. 109, pp. 33-48.

GRIMM, Jacob (1864). «Über den Ursprung der Sprache», Reden und Abhandlungen (= Kleinere Schriften, 1. Band). Berlin, Ferd. Dümmler's Verlagsbuchhandlung.

GÜNTHER, Hartmut (1985). «Probleme beim Verschriften der Muttersprache - Otfrid von Weißenburg und die «lingua theotiscam", dans Zeitschrift für Literaturwissenschaft und Linguistik, vol. 59, pp. 36-54.

KARTSCHOKE, Dieter (1975). Bibeldichtung - Studien zur Geschichte der epischen Bibelparaphrase von Juvencus bis Otfrid von Weißenburg. Munich, Wilhelm Fink Verlag.

KLEIBER, Wolfgang (éd. 1978). Otfrid von Weißenburg. Darmstadt, Wissenschaftliche Buchgesellschaft, Wege der Forschung 419.

Magoun Jr., Francis P. (1943). "Otfrid's «Ad Liutbertum», dans Publications of the Modern Language Association of America, vol. 58, pp. 869-890.

Michel, Paul et Alexander Schwarz (1978). Unz in obanentig - Aus der Werkstatt der karolingischen Exegeten Alcuin, Erkanbert und Otfrid von Weißenburg. Bonn, Bouvier.

Patzlaff, Rainer (1975). Otfrid von Weißenburg und die mittelalterliche versus-Tradition. Tübingen, Niemeyer.

PAul, Hermann et Heinz Stolte (1962, 1re éd. 1949). Kurze deutsche Grammatik. Tübingen, Niemeyer.

RädLE, Fidel (1974). «Otfrids Brief an Liutbert», dans SCHMIDT, ErnstJoachim, éd. Kritische Bewahrung - Beiträge zur deutschen Philologie, Festschrift für Werner Schröder. Berlin, Erich Schmidt Verlag, pp. 213-240.

Raynaud, Franziska (1982). Histoire de la langue allemande. Paris, Presses Universitaires de France, «Que sais-je?», 1952. 
Rerss, Katharina et Hans J. Vermeer (1984). Grundlegung einer allgemeinen Translationstheorie. Tübingen, Niemeyer, Linguistische Arbeiten 147.

REISS, Katharina (1984). «Adäquatheit und Äquivalenz», dans WILSS, Wolfram et Gisela Thome, éd. Die Theorie des Übersetzens und ihr Aufschlußwert für die Übersetzungs- und Dolmetschdidaktik. Tübingen, Gunter Narr, Tübinger Beiträge zur Linguistik 247, pp. 80-89.

SCHLOSSER, Horst Dieter (1970). Althochdeutsche Literatur - Ausgewählte Texte mit Übertragungen und Anmerkungen. Francfort, Fischer Bücherei 6036.

SCHRÖBLER, Ingeborg (1975). «Syntax», dans PAUL, Hermann, Hugo Moser et Ingeborg Schröbler. Mittelhochdeutsche Grammatik. Tübingen, Niemeyer.

SCHRÖDER, Werner (1987). «Otfrid von Weißenburg», dans RUH, Kurt, éd. Die deutsche Literatur des Mittelalters - Verfasserlexikon, vol. 7, 1987, col. 172-193, Berlin, De Gruyter.

SchwAB, Ute (1980). "Die "cornua crucis" und «thes kruces horn"», dans Zeitschrift für deutsches Altertum, vol. 109, pp. 1-33.

SONDEREGGER, Stefan (1974). Althochdeutsche Sprache und Literatur. Berlin, De Gruyter.

STÖRIG, Hans Joachim (1969). Das Problem des Übersetzens. Darmstadt, Wissenschaftliche Buchgesellschaft, Wege der Forschung VIII.

Wunder, Dieter (1975). Der Nebensatz bei Otfrid - Untersuchungen zur Syntax des deutschen Nebensatzes. Heidelberg, Winter.

ZEMB, Jean-Marie (1968). Les Structures logiques de la proposition allemande. Paris, O.C.D.L. 